\title{
Public Transport Assistant for Visually Impaired (PuTAVI)
}

\author{
Sathvik N Prasad ${ }^{1}$, Shrikanth S Hegde ${ }^{1}$, Subodh B Hegde ${ }^{1}$, Vishwas H P ${ }^{1}$, Sreekar $\mathbf{V}^{1}$, Roopa J $^{2}$, \\ Govinda Raju $\mathbf{M}^{2}$
}

Student, ECE, RV College of Engineering, RV Vidyaniketan Post, Mysore Road, Bangalore, India ${ }^{1}$

Assistant Professor, ECE, RV College of Engineering, RV Vidyaniketan Post, Mysore Road, Bangalore, India ${ }^{2}$

\begin{abstract}
Visually challenged people rely on support for managing their routine, be it in crossing busy roads, climbing steps or boarding buses. Applying technology to make these people self-reliant and independent is a social responsibility. It is high time that a novel technological solution be found. The objective of this project is to provide technologically supported audio guidance system to visually challenged people, with respect to obstacle and bus identification on an MSP430 platform making use of ultrasonic sensors, RF Transmitter and Receiver. The system is wearable by the user and user friendly in its interface.
\end{abstract}

Keywords: Signal Conditioning; Audio playback; Obstacle Detection System; Bus Identification System; Ultrasonic sensors

\section{INTRODUCTION}

This project is oriented towards helping visually impaired Since a blind person is not able to see the display panel or people with appropriate voice commands that are played control buttons, hence the device should be easily through an earphone. The proposed design is the controllable. Moreover, the ETA device should be of lowarchitecture of a new concept we have christened cost so as to be affordable by a common man. Recent "PuTAVI". The project aims at making navigation and trends showcases a wide range of research work initiated commutation smoother and easier for these people by in the direction of designing high end and electronically administering a sub system which gives audio information advanced robotic guides which can not only provide safe about the route numbers of local transport (bus in this navigation but these aids have intelligent interfaces that case). The advantage of the system lies in the fact that it provide simple physical and cognitive assistance such as can prove to be a cost effective solution to millions of obstacle avoidance and public transport assistance.

blind people worldwide. The proposed combination of various working units as shown in Figure1, makes a realtime system that monitors position of the user and provides appropriate voice information, making navigation more safe and secure.

\section{A .Technical Background}

Recently there has been a lot of Electronic Travel Aids (ETA), designed and devised to help the blind people to navigate safely and independently. The most important factors, which enable blind users to accept these devices readily, are portability, low cost, and above all simplicity of controls. Hence, ETA device should be small in size and lightweight for portability.

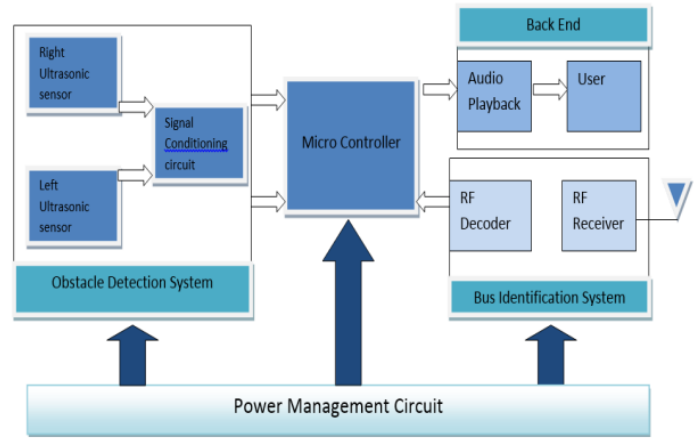

Figure 1: Top Level Block Diagram

\section{B. Proposed Solution}

In comparison with other technologies many blind guidance systems use ultrasound because of its immunity to the environmental noise. Another reason why ultrasonic is popular is this technology is relatively inexpensive, and also the ultrasound emitters and detectors are small enough to be carried without the need for complex circuitry. Apart from the conventional navigation systems, a blind aid system can be provided a new dimension of Real-time-assistance and artificial vision along with dedicated obstacle detection circuitry.

\section{Organization of the Paper}

Section 2 of the paper substantiates the proposed solution provided. It throws light on the major hardware and software sub-systems. Section 3 elucidates the hardware implementation and the software logic. Section 4 of the paper provides the results of simulations. Section 5 deals with the scope for improvement in the project.

\section{PROPOSED SOLUTION}

Figure 2 represents the System Level Diagram of PuTAVI. Since a prototype has been presented the assumptions made 
for the input is from 2 buses and hence a unique audio is To start measurement, a pulse of high(5V) for 10 us is sent played for each of the buses.

to TRIG pin of SR04, this will initiate the sensor which transmits 8 cycles of ultrasonic burst at $40 \mathrm{Khz}$ and wait

The main constraint in the system was to consider that a transmitter is present on the bus which has to be detected.

The main hardware parts in the circuit are as follows:

\begin{tabular}{ll} 
- & Sensors \\
- & Signal Conditioning \\
- & Microcontroller \\
- & Audio Playback IC \\
- & RF Decoder and receiver \\
\hline & User Controlled Switch
\end{tabular}

The software in the system mainly considers the code which governs the working of the subsystems. The software used in developing the above system are:

- $\quad$ Code Composer Studio, Version 5.2.1

- Termite Serial Monitor

- $\quad$ TINA TI Version 9

The analog subsystem is a $5 \mathrm{~V}$ to $3.3 \mathrm{~V}$ converter. This converter helps in conditioning the sensor output in order to provide input to MSP430, for processing.



Figure 2: System Level Diagram

\section{IMPLEMENTATION}

\section{A. Hardware Implementation}

The overall hardware mainly is classified into powersupply unit, sensor system, signal conditioning, microcontroller, R. F. transmission and reception along with encoder and decoder, and the audio logging and playback unit.

\section{- $\quad$ Power Supply unit:}

The power-supply unit consists of 2 regulators namely LM3940 and UA7805. The 5 volt output got after the regulation is used to power the sensor unit, signal conditioning, RF and the audio sub-system. The 3.3 volt output is used to power the msp430 microcontroller.

\section{- Sensor unit:}

The Sensor unit consists of 2 ultrasonic sensors (HCSR04). for the reflected ultrasonic burst. When the sensor detects ultrasonic reflection from receiver, the echo pin is set to $5 \mathrm{~V}$ and the duration of it is proportional to the distance of the reflecting object as shown in Figure 3.

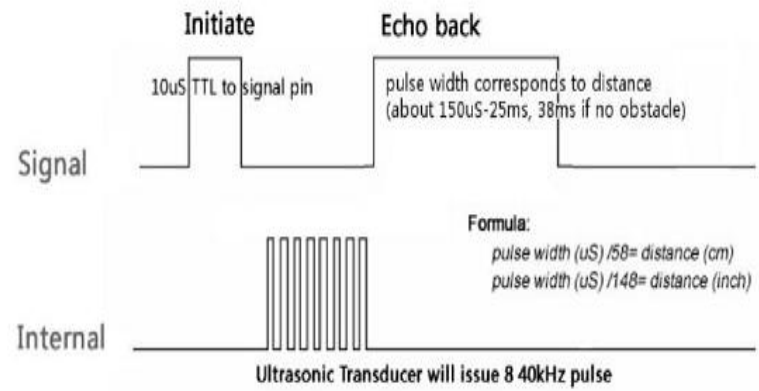

Figure 3: Timing waveform of HCSR-04

According to calibrations of the sensor the distance in $\mathrm{cm}$ is equal to the $1 / 58$ times the durarion for which the echo pin is set high.

\section{- Signal Conditioning unit:}

vout $=a \times$ vin $+b$

Design Requirements:

$\mathrm{V}_{\text {out }}=3.3 \mathrm{~V}$ when $\mathrm{V}_{\text {in }}=5 \mathrm{~V}$

$\mathrm{V}_{\text {out }}=0 \mathrm{~V}$ when $\mathrm{V}_{\text {in }}=0 \mathrm{~V}$

Substituting in Eqn(1)

We get $a=3.3 / 5 ; b=0$; Designing $a=3.3 / 5$ considering

Standard resistance values and tolerances:

$\mathrm{a}=\mathrm{R} 1 /(\mathrm{R} 1+\mathrm{R} 2)$

$\mathrm{R} 1=330 \mathrm{~K} \Omega$

$\mathrm{R} 2=170 \mathrm{~K} \Omega \sim 169.7 \mathrm{~K} \Omega$

$(150 \mathrm{~K} \Omega+15 \mathrm{~K} \Omega+4.7 \mathrm{~K} \Omega$; standard values)

The circuit designed is shown in Figure 4

Selection of op-amp:

Since we need a unipolar swing in the output of the circuit; a single supply op-amp circuit has been designed for the above use. The OPA4277PA was chosen in order to optimise the space as the package consists of 4 op-amps with improved noise immunity and a good slew rate.

Since the pulse width of the sensor is as less as 10us the op-amp used to shift the level should have a good slew rate. The specifications of OPA4277PA just proved perfect for the above application.

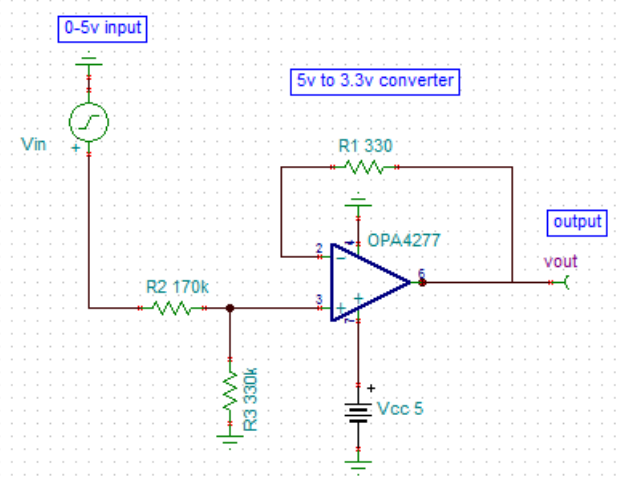

Figure 4: OpAmp 4277 5V to 3.3V converter for Signal Conditioning 


\section{- RF unit:}

Encoder: The $2^{12}$ series of encoders begin a 4 word transmission cycle upon receipt of a transmission enable.

The HT12E and the transmitter circuit is as shown in figure 5 .

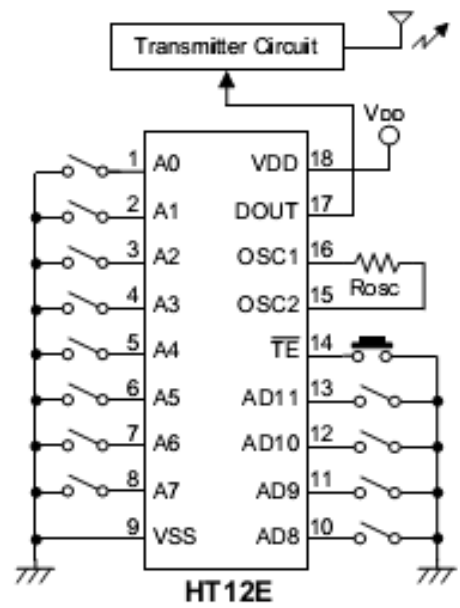

Figure 5: HT12E encoder circuit with RF Transmitter

This cycle repeats itself as long as the transmission enable is low. Once the transmission enable returns high the encoder output completes its final cycle and then stops. The transmission timing is as shown in Figure 6.

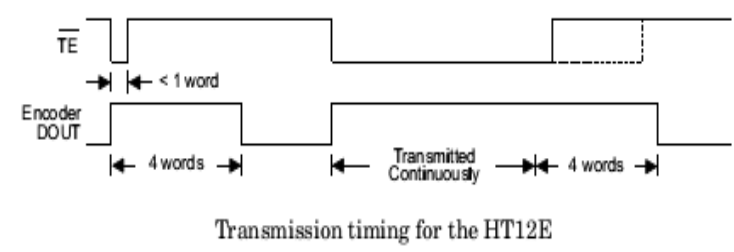

Figure 6: Timing waveform of RF Transmitter

\section{Decoder:}

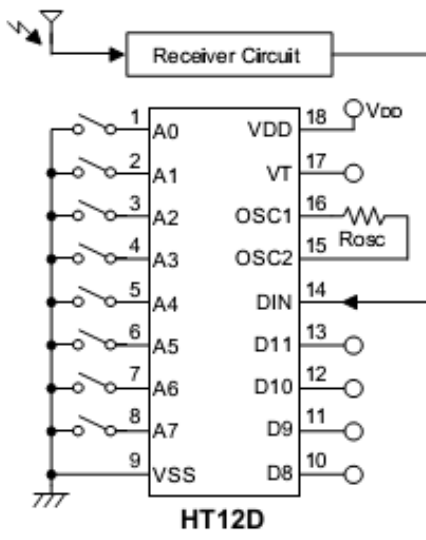

Figure 7: Receiver circuit with Decoder
The $2^{12}$ series of decoders provides various combinations of addresses and data pins in different packages so as to pair with the $2^{12}$ series of encoders.

\section{- $\quad$ Microcontroller MSP430G2553}

The Microcontroller used in the project is MSP430G2553.The MC is mainly used to synchronize all the sub-systems. The function served by the microcontroller is mainly to trigger the ultrasonic sensors and then wait for the echo pulse from the sensor. At first a user controlled switch is provided as an input to the microcontroller. The user can switch between obstacle detection and bus identification mode. Based on this decision the MC controls the audio feedback system with corresponding and relevant messages as previously logged.

\section{- $\quad$ Audio Playback System(APR33A3)}

The Audio playback system gives appropriate voice commands to the VI person through earphone. The system consists of a powerful 16 bit audio processor (APR33A3) which has a built in high resolution ADC and DAC. The system also has an audio recording microphone amplifier through which the user can record the voice messages at 8 different channels. In this project we used only four voice commands left, right, busnumber 1 and bus number 2 which are recorded at 4 different channels. The recorded voice can be played back by selecting respective channels through MSP430 micro-controller.

The final product is made wearable and is compact. It is portable and light to carry around for outdoor navigation.

The finished product PuTAVI is as shown in the Figure 8.

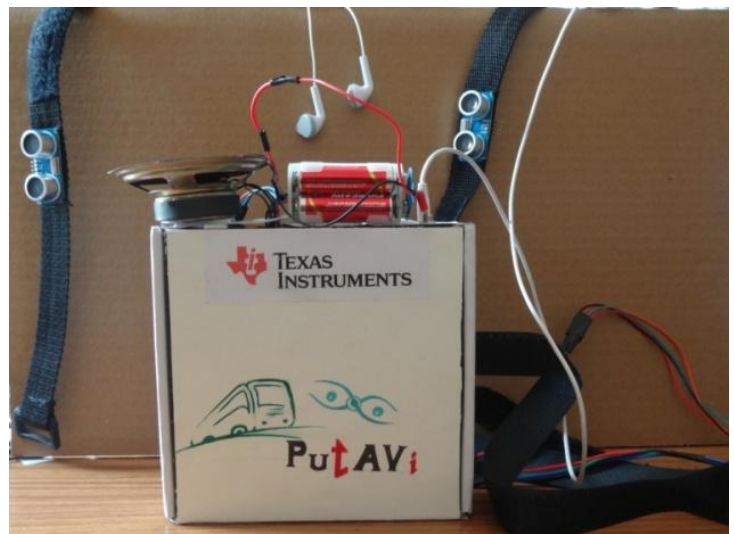

Figure 8: Finished Product PuTAVI

\section{B. Software Implementation}

The logic flow of the circuit is shown in the following block diagrams.

The system level flowchart is as shown in Figure 9. 


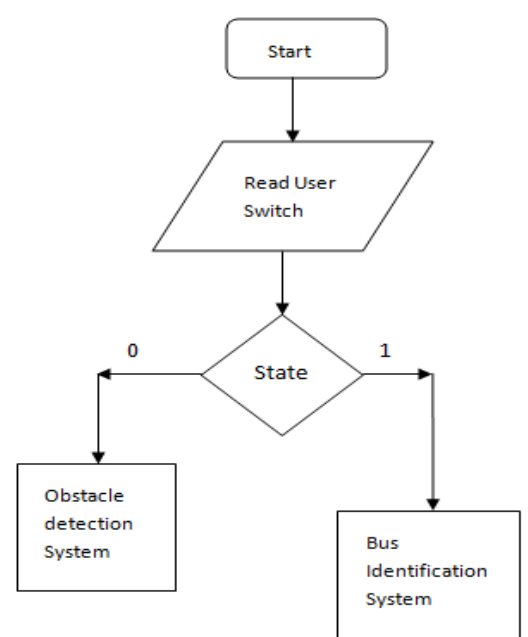

Figure 9: System Flow chart

The user is given a switch to choose between the modes. Two modes have been provided:

- $\quad$ Obstacle Detection System

- $\quad$ Bus Identification System

Both the systems are controlled by MSP430 and share a common output through the audio feedback system.

The logic flow of the Obstacle Detection System is as shown in the flowchart in Figure 10.

The device is calibrated such that the obstacle is sensed only when it is at a particular distance from the user.

The decision making in all the stages of logic flow is done by the microcontroller MSP430.

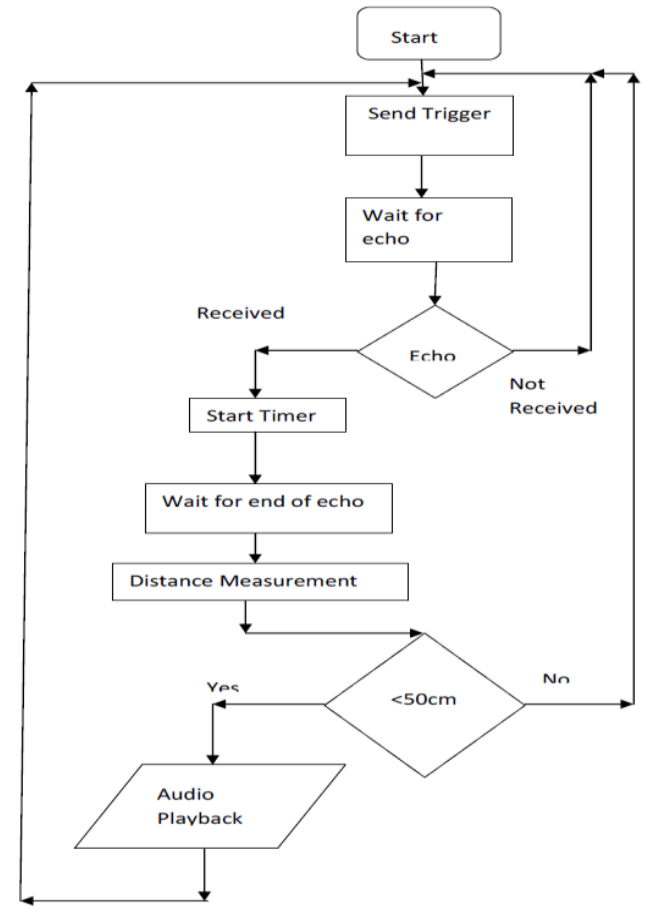

Figure 10: Obstacle Detection System flowchart
The logic flow of the Bus Identification System is as shown in Figure 11.The bus identification system is a pair of RF Transmitter and Receiver. When the bus approaches the bus stop the RF transmitter sends a message which is picked up by the receiver and decoded. This information is given to the user via audio feedback. The user decides accordingly. The merit of the decoder is that $2^{12}$ buses can be detected.

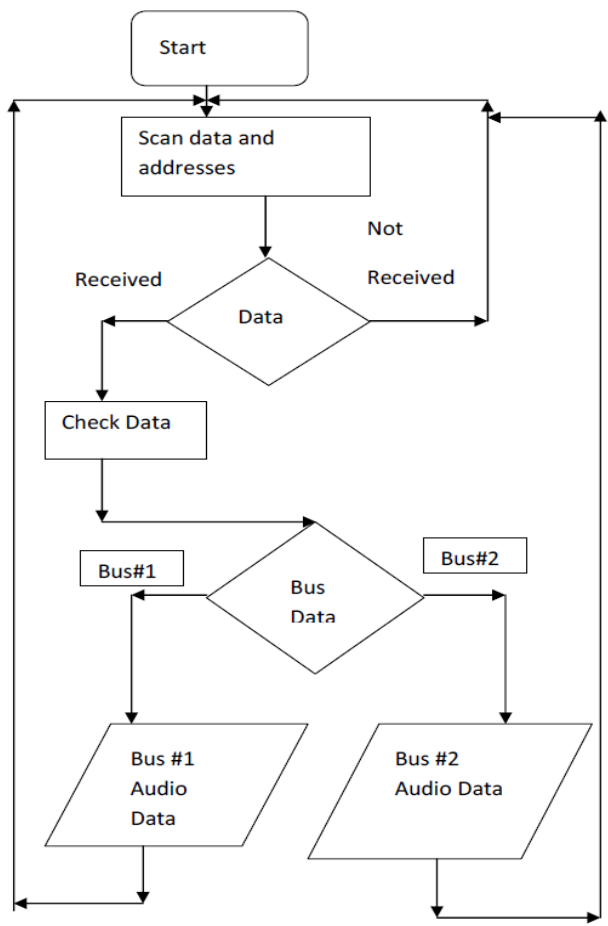

Figure 11: Bus Identification System flowchart

\section{RESULTS}

The simulation result of the signal conditioning circuit designed using OPA4277PA is shown below. The purpose of the circuit is to level shift the signal from $5 \mathrm{~V}$ to 3.3V.The result is shown in Figure 12.

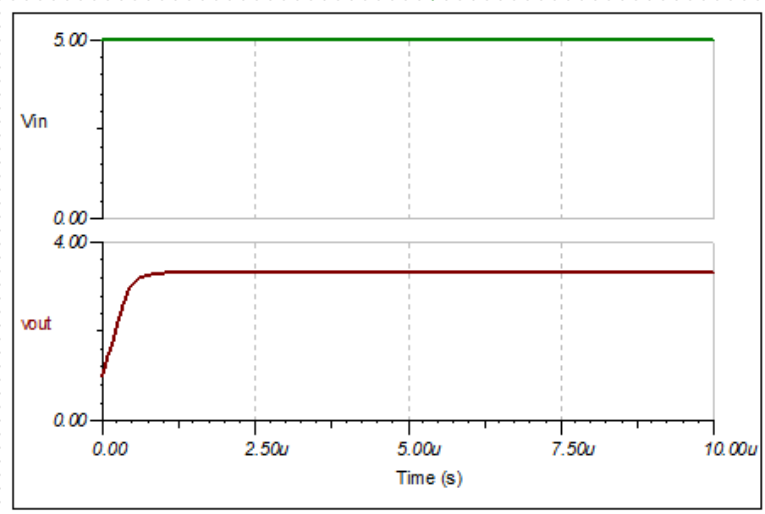

Figure 12: Simulation results of level shifter

The ultrasonic sensors were calibrated using Termite 2.9. The circuit is calibrated for an optimum distance. The screen grab of the above calibration is as shown in Figure 13. 
International Journal of Advanced Research in Computer and Communication Engineering Vol. 3, Issue 11, November 2014

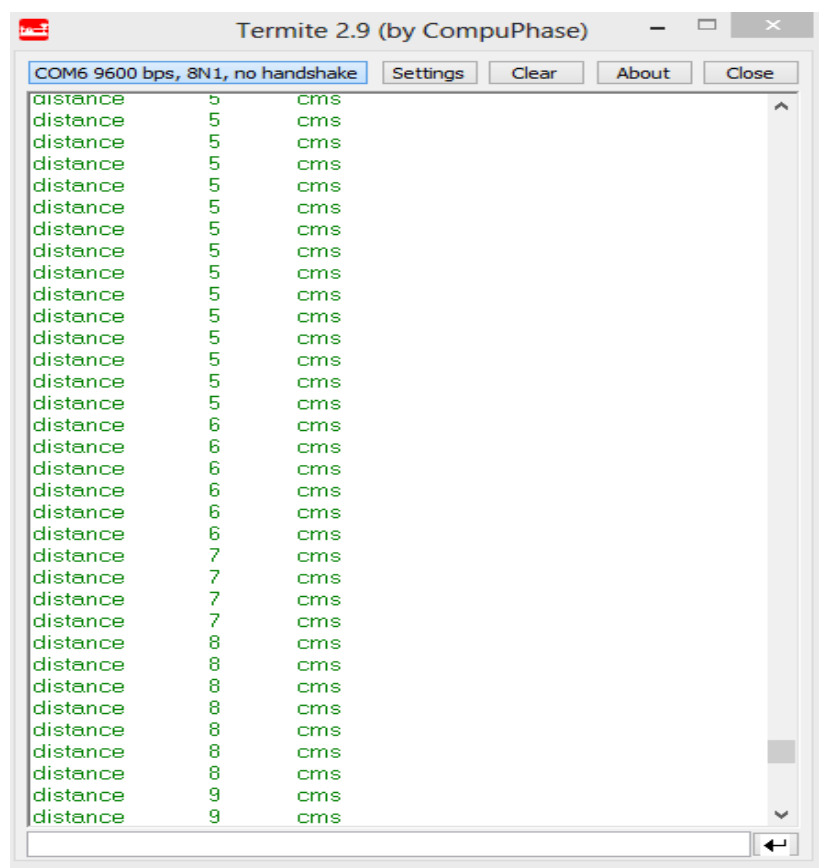

Figure 13: Calibration and testing of Ultrasonic sensor

\section{HCSR-04}

\section{CONCLUSIONS}

The system provides an integrated solution of both obstacle detection and public transport assistance. The main objective of the system was to make the system compact, low power and wearable, which has been accomplished. Since a prototype of the product is presented, it has been limited to an extent of 2 buses. This can be extended to any form of transport and number.

The user can select the mode of his choice i.e., between obstacle detection and public transport assistance. The overall product is compact and low power, and hence can be carried around with minimum effort and least concern regarding powering of the circuit.

When low cost isn't a priority the system can be made robust with the use of a GPS module. The audio playback can be made clearer and the storage capability can be increased with the help of SD card. The use of SD card was intended but the logging and playback was too slow. We had two options on hand; that was to change the platform or to change the approach for audio playback. We went ahead with the use of audio playback ICAPR33A3; this can be done better with the help of a SD card and a more improved platform with enhanced capabilities.

\section{REFERENCES}

[1]. Amit Kumar, RushaPatra, M. Manjunatha, J.Mukhopadhyay and A. K. Majumdar An electronic travel aid for navigation of visually impaired Communication Systems and Networks (COMSNETS), 2011 Third International conference on 4-8 Jan 2011.

[2]. Calder, David J.; Curtin .An obstacle signaling system for the blind ,Digital Ecosystems and Technologies Conference (DEST), 2011Proceedings of the 5th IEEE International,Conference on 30 June 2011. 